\title{
Erratum to: Designing a Phosphorus Removal Structure
}

\section{Erratum to:}

Chapter 6 in: C. Penn, J.M. Bowen, Design and Construction of Phosphorus Removal Structures for Improving Water Quality, DOI 10.1007/978-3-319-58658-8_6

The original version of this chapter was inadvertently published with an incorrect value (140) in the footnote under the table 6.3. The correct value is updated as " 242 ". 